\title{
Locating an invisible goal in a water maze requires at least two landmarks
}

\author{
JOSÉ PRADOS and JOSEP B. TROBALON \\ University of Barcelona, Barcelona, Spain
}

\begin{abstract}
In three experiments, rats were trained in a spatial task in a swimming pool similar to that used by Morris (1981), but surrounded by black curtains that provided a great level of control over the landmarks that defined the location of the goal. Experiment 1 showed that rats can learn to find an invisible platform in a fixed location relative to four landmarks in a very homogeneous environment. Experiments 2 and 3 demonstrated that the animals need two landmarks to recover the information about the environment stored in their memory as a cognitive map.
\end{abstract}

Tolman (1948) concluded that rats organize their knowledge of the environment in cognitive representations or maps. These cognitive maps are representations of space relations between different places, and they permit the animals to plan and follow new routes when moving from one place to another.

From a given location, an animal can locate a goal using the information it can obtain from the main landmarks present in its environment. Tolman did not distinguish this space learning strategy from other strategies that require, for example, the mere approximation to a specific cue that defined by itself the location of the goal. This confusion, and the difficulty in proving the existence of cognitive maps in the traditional experiments about space learning, was the reason that the concept of cognitive maps was put aside.

Until the 1970s, the research about space learning was focused on the "place-versus-response" question: whether the animals learned a T-maze using a place strategy, like Tolman's theory supposed (Tolman, 1932, 1948), or using a response strategy, like Hull's theory suggested (Hull, 1943). For example, Restle (1957) concluded that, in the learning of a space discrimination task in a T-maze, we can find the two strategies, but one of them would be dominant in function of the environmental conditions in which the training is covered. Thus, the response strategy will be dominant when the training is carried out in a homogeneous environment with weak illumination, whereas the place strategy will be dominant when the animals are trained in a heterogeneous environment and in conditions of good illumination. In the same work, Restle argued that the concept of cognitive map is unnecessary to explain space learning, since the animals are able to locate

The research reported here was supported by Grant PB93-0739 from the Spanish Ministerio de Educación y Ciencia. The authors are indebted to Robert Boakes for comments on an earlier draft and to Pablo Guzmán for helping to build the apparatus. Correspondence should be addressed to J. B. Trobalon, Universitat de Barcelona, Departament de Psicologia Bàsica, Passeig de la Vall d'Hebron, 171, 08035-Barcelona, Spain (e-mail: jbtrobalon@psi.ub.es). a goal perfectly on the basis of a simple associative learning process.

O'Keefe and Nadel (1978) recovered the concept of cognitive map and offered a formal definition: A cognitive map is a storage in long-term memory of the representation of different places connected by distances and directions. The idea of the strategy of learning maps (cartographic strategy, as they referred to it) was not new; however, the conception of the cartographic strategy as a different kind of learning, independent of any other kind of learning, was new. Thus, they proposed a new taxonomy of space learning, distinguishing the cartographic strategy from other associative strategies traditionally referred to in order to explain the behavior of rats in mazes, such as the learning of response sequences and place strategy - both included in a new category, the taxon strategies - which they renamed orientation and guide learning, respectively.

Morris (1981) designed a task that required the animals to use a cognitive map. Morris used a circular pool full of opaque water. Inside the pool, $1 \mathrm{~cm}$ under the water level, there was a platform in a fixed location that was absolutely invisible to the animals. He trained the rats in an escape task: The animals had to escape from the water by swimming to the platform. In the beginning, the rats swam at random; however, after a few trainings, they began to swim directly from whatever starting point toward the invisible platform, which was always in the same position in respect to the landmarks that surrounded it. These results contributed favorable evidence to the existence and use of a cognitive map. The precise behavior of the animals in Morris's pool is difficult to explain in terms of orientation learning, because, in each training, the starting point was changed, or in terms of guide learning, because there was not any cue that defined by itself the location of the invisible platform. Thus, according to Morris, the best explanation for the behavior of his animals was the cognitive map.

One of the characteristics that give the cognitive map great flexibility and adaptive capacity, such as was defined by O'Keefe and Nadel (1978), is that it contains redun- 
dant information: None of its landmarks that define the position of a goal is indispensable for its location. This hypothesis was tested by Chamizo, Sterio, and Mackintosh (1985), who found that some animals, trained in a three-arm maze under conditions that elicited the cartographic strategy, maintained their performance level in a space discrimination task, even though some landmarks were removed from the experimental room.

Our work was carried out in a circular pool very similar to the one used by Morris (1981), but surrounded by black curtains that formed a homogeneous enclosure. Inside the curtains, we placed some objects as landmarks hanging from the ceiling. In order to ensure that the rats used these landmarks, rather than any inadvertently remaining static room cues, to locate the platform, the landmarks and platform were rotated, with respect to the room, between each trial. In this way, it was easy to control the landmarks that defined the location of the invisible platform. Our purpose was to explore the point at which the animals were dependent on the number of landmarks to maintain a good performance level in a space learning task working under conditions similar to those in the Morris experiments.

\section{EXPERIMENT 1}

The aim of Experiment 1 was simply to find whether, under the general conditions used in this series, rats can learn to use four landmarks to identify the position of the hidden platform. To this end, a group for whom there was a consistent relationship between the landmark array and the platform (place group) was compared with a group for whom this relationship was varied during training (varied group).

\section{Method}

Subjects and Apparatus. The subjects were 14 Long-Evans rats ( 8 males and 6 females) between 3 and 4 months old and experimentally naive. They were housed in pairs on ad-lib conditions of food and water. They were subjected to a 12:12-h light:dark cycle (lights on $0800-2000$ ), and the experiment was carried out between 1400 and 2000 . These conditions were used in all the experiments in the present study. A large circular pool $(158 \mathrm{~cm}$ in diameter and $65 \mathrm{~cm}$ deep) was used, as in Morris (1981) (see Figure 1). It was filled to a depth of $49 \mathrm{~cm}$ with water that was made opaque by adding $750 \mathrm{ml}$ of polystyrene latex, a nontoxic substance that does not spoil. A constant temperature of $21^{\circ} \mathrm{C} \pm 1^{\circ}$ was maintained. The pool was situated in the middle of a large room, mounted on a wooden platform $43 \mathrm{~cm}$ above the floor. A circular platform $(11 \mathrm{~cm}$ in diameter) made of transparent Perspex was mounted on a pillar and base and was placed in one quadrant of the pool, $38 \mathrm{~cm}$ from the side, with its top $1 \mathrm{~cm}$ below the surface of the water. The pool was surrounded by black curtains reaching from ceiling to floor and forming a circular enclosure $240 \mathrm{~cm}$ in diameter. In the center of the enclosure and $175 \mathrm{~cm}$ above the center of the pool was a lighting system made up of three low-consumption light bulbs ( $70 \mathrm{~W}$ each). A videocamera was mounted in the center of this system with a wide-angle lens that transferred the image of the pool to a tracking system with computerized registration, which consisted of a realtime image processor (Video-Track 512) and computer (PC 286) situated in an adjacent room.

The four landmarks were as follows: (A) a white die cube $(25 \times$ $25 \times 25 \mathrm{~cm}$ ) with a black dot in the center of each of its six sides; (B) a black transistor with a radio station tuned in (variable noise, 60-70 dB); (C) a round white balloon $35 \mathrm{~cm}$ in diameter; (D) a white transistor with a constant noise $(60 \mathrm{~dB})$. The location of the platform was between Cue A and Cue B. The landmarks could be suspended from the ceiling, $35 \mathrm{~cm}$ above the surface of the water, with their midlines directly above the wall of the pool. Their loca-

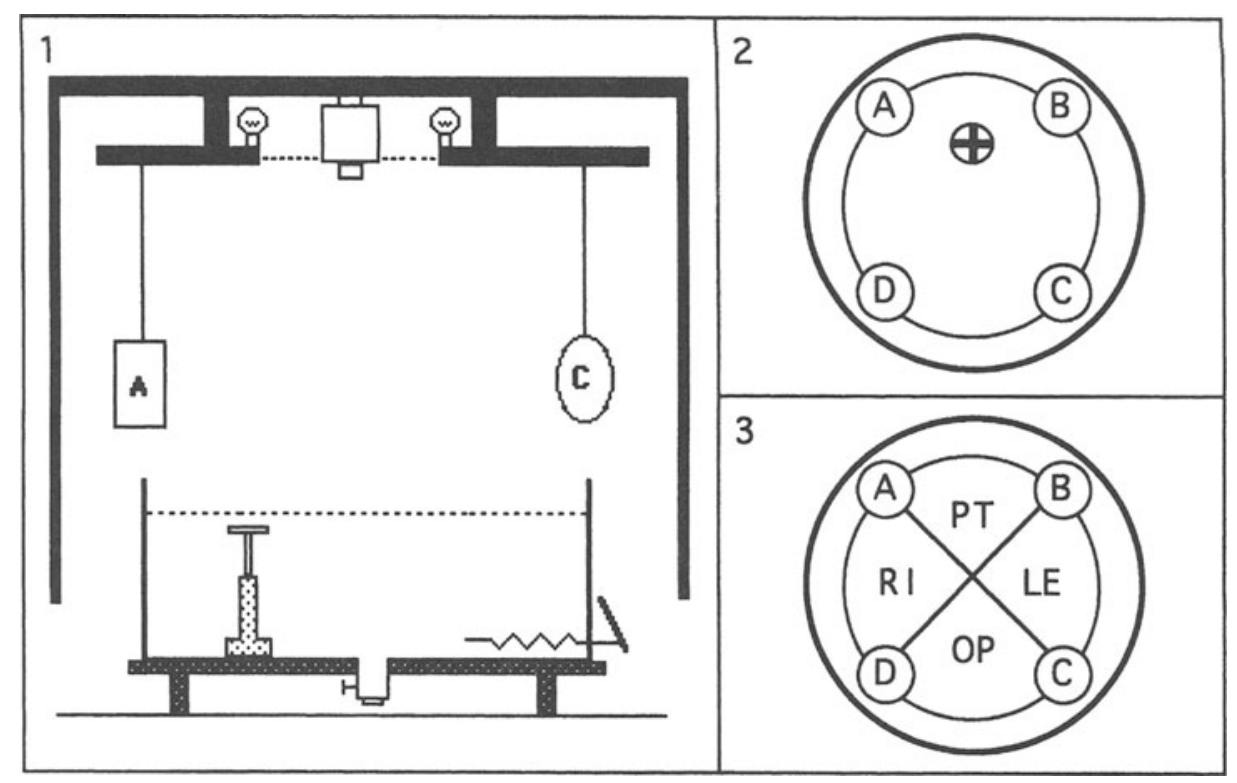

Figure 1. (1) Diagram illustrating the circular pool used in the present study. (2) A schematic representation of the pool, with the landmarks and the platform, during the training. (3) A schematic representation of the pool, with the landmarks and the four areas designed for the test. 
tions relative to the platform are shown in Figure 1. The platform and the entire false ceiling, with these landmarks suspended, could be rotated from trial to trial.

Procedure. The two groups ( $n s=7)$, Group Place and Group Varied, were matched for age and gender. In the first phase (pretraining), identical for all subjects, each animal could swim freely in the pool for 3 min on 2 successive days, without the presence of the platform or any landmarks. In the second phase (training), the four landmarks and the invisible platform were introduced. The animals were trained in an escape task for 8 days at a rate of eight trials daily. At the beginning of each training trial, the subject was introduced into the pool from one of four starting points (face to the wall) and was allowed to swim freely for a maximum of $3 \mathrm{~min}$, until it found the platform. There, the animal remained for $1 \mathrm{~min}$. For each trial, the landmarks and platform were rotated $90^{\circ}$ in a counterclockwise direction to ensure that there were no other cues affecting the performance of the rats. The interval between trials was approximately $5 \mathrm{~min}$. For the subjects in Group Place, the platform always remained in a fixed position in relation to the landmarks; for the subjects in Group Varied, the platform position varied between four different positions from trial to trial. Both the escape time of each subject (defined as the time spent by each animal from the moment it was deposited into the pool until the moment it found the platform) and the distance covered were recorded for each trial.

The third phase (test) was identical for both groups. All animals were given two trials: one of retraining (identical to the training trials) and one of testing. In the test trial, the platform was removed from the pool, and the subject could swim freely for $2 \mathrm{~min}$. The computer image of the swimming pool was divided into four quadrants that corresponded to those for Group Place: the area in which the platform was located during the training (PT), its two adjacent areas-area right (RI) and area left (LE)-and the area opposite (OP). The swimming time and the distance covered by the animals in each quadrant were measured.

\section{Results and Discussion}

Figure 2 shows the mean escape time and the mean distance covered by the subjects of the two groups in the training phase. An analysis of variance (ANOVA) of repeated measures demonstrated that there were no significant differences between Groups Place and Varied for the mean time spent to escape, and the days factor was significant $[F(7,84)=18.309, p<.01]$. With respect to the mean distance covered in each training session, there were significant differences between groups $[F(1,7)=$ $7.565, p<.05]$ and between days $[F(7,84)=26.069, p<$ $.01]$, and there was a significant groups $\times$ days interaction $[F(1,84)=2.331, p<.05]$.

Figure 3 shows the mean time spent and the mean distance covered in Area PT versus Areas RI, LE, and OP on the test trial. An ANOVA demonstrated that there were significant differences in the time spent between groups $[F(1,12)=4.404, p=.05]$ and in the areas factor (Area PT vs. Areas RI, LE, and OP) $[F(1,12)=6.931$, $p<.01]$, and there was a significant groups $\times$ areas interaction $[F(1,12)=4.539, p<.05]$. The analysis of the simple effects showed that differences existed between groups in the areas $(p=.05)$, with the difference in time spent by the subjects of Group Place in Area PT greater than that in the other three areas $[F(1,12)=11.343, p<$ $.01]$; this difference was not significant for Group Varied. With regard to the distance covered in the different areas, there was no significant difference between groups, although the areas factor was significant $[F(1,12)=5.070$, $p<.05]$, as was the groups $\times$ areas interaction $[F(1,12)=$ $5.372, p<.05]$. The analysis of simple effects showed significant differences between the distance covered by the subjects of Group Place in Area PT, relative to in the other three areas $[F(1,12)=10.440, p<.01]$; this difference was not significant for the subjects of Group Varied.

Although the results of the training phase suggest that the animals of Group Place learned to find the goal using the information provided by the landmarks around its location in the pool, we still cannot affirm, on the basis of these data, that these animals used a cognitive map strategy. However, the results of the test phase are very clear: The subjects of Group Place spent more time and covered a greater distance in Area PT (which corresponds to the quadrant where they found the platform during the training) than in any of the other three areas, whereas the
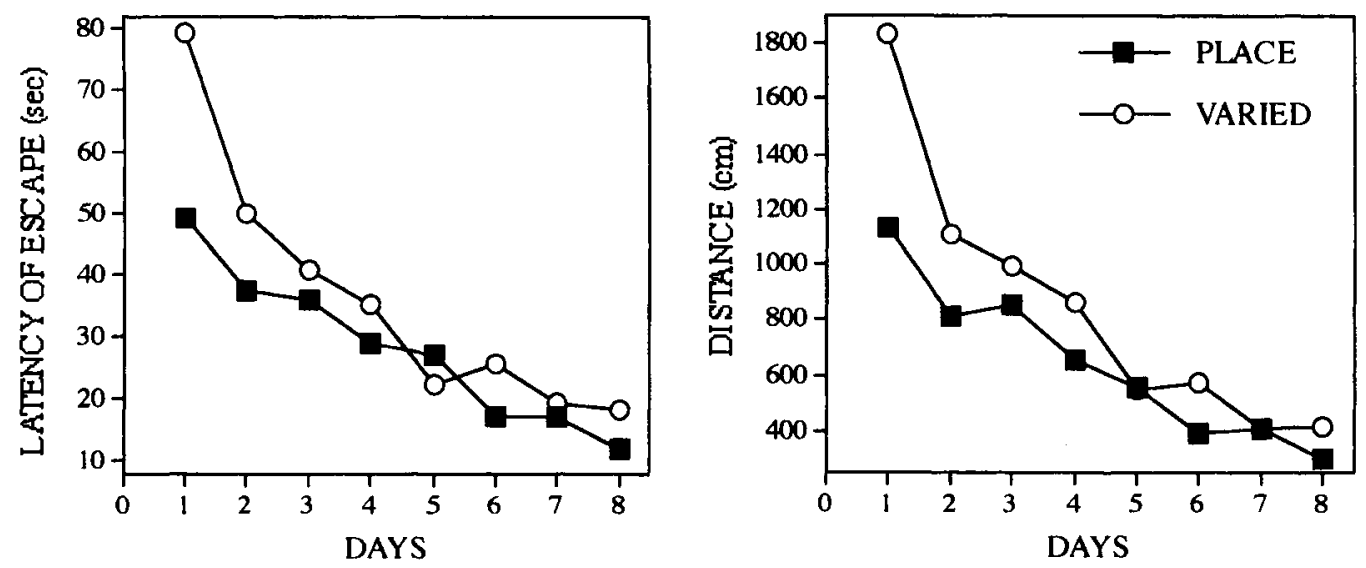

Figure 2. For Experiment 1, mean escape time (left) and mean distance covered (right) by Groups Place and Varied in the eight training sessions. 

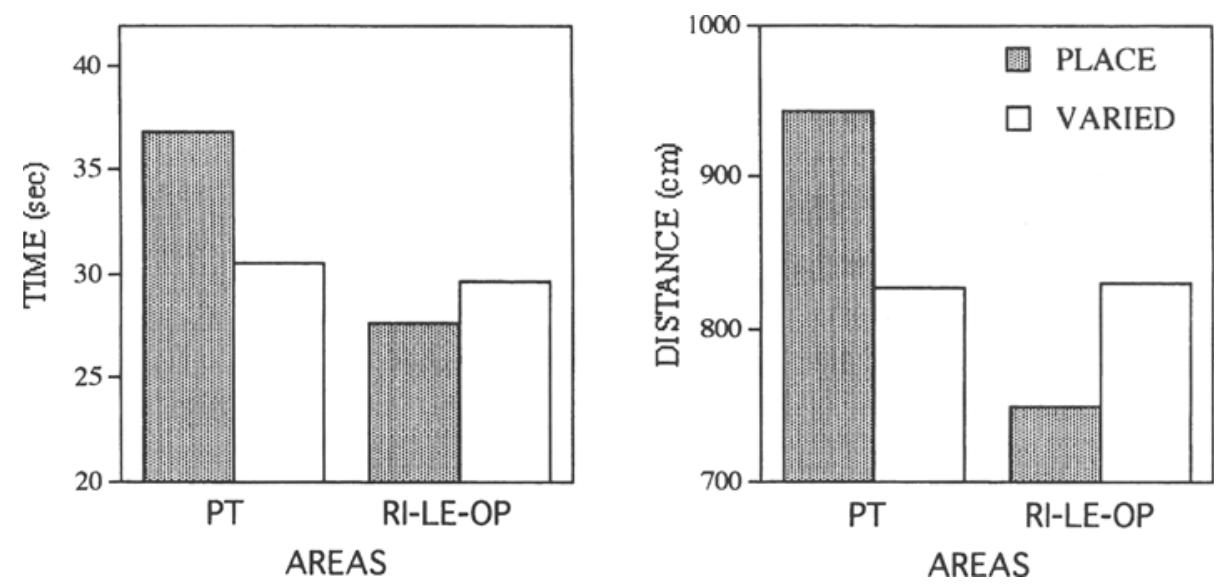

Figure 3. For the test phase of Experiment 1, mean time spent (left) and mean distance covered (right) by Groups Place and Varied for the area in which the platform was located during the training (PT) and the other three areas (RI-LE-OP).

animals of Group Varied spent an equal amount of time and covered an equal distance in the different areas. The subjects of Group Place, therefore, learned to locate the invisible platform on the basis of the information from the different landmarks.

\section{EXPERIMENT 2}

The results of Experiment 1 confirmed that, under the present conditions, rats can learn the location of an invisible platform by using distal cues as landmarks. In other preparations for studying spatial learning, evidence has been obtained suggesting that there is considerable redundancy in the information that rats encode about their environment. Thus, using a T-maze, O'Keefe and Conway (1978) found that, following training with four landmarks, performance remained above chance when many of these landmarks were removed. A similar result has been obtained using the radial maze. For example, Chamizo et al. (1985) trained animals with multiple landmarks and found that, on test, performance remained above chance although some landmarks were removed. The aim of Experiment 2 was to see whether this result would be found using the water maze.

\section{Method}

Subjects and Apparatus. The subjects were 7 male Long-Evans rats, 6 months old at the beginning of the experiment and experimentally naive. The animals were trained in the circular pool used in Experiment 1, and the landmarks used this time were similar: (A) a fixed 40-W light; (B) a black transistor with a radio station tuned in (variable noise, $60-70 \mathrm{~dB}$ ); (C) a white round balloon, $35 \mathrm{~cm}$ in diameter; (D) a white transistor with a constant noise (60 dB). In other words, Cue A was different from that of Experiment 1, but the other cues remained as before. The location of the platform was between Cue $A$ and Cue B.

Procedure. The training procedure consisted of the same eight sessions as were used for Group Place in Experiment 1. The test phase consisted of 3 test days, each containing four trials: one re- training, one test trial, and two more of retraining. For the test trials, the procedure was the same as in Experiment 1 . The four landmarks that had been present during the training remained in Test 1 . However, in Test 2, two of the landmarks were removed. The two landmarks present were different for each rat. Five combinations of landmarks were used (for each of 5 rats, the combinations were $A B$, $\mathrm{AC}, \mathrm{BC}, \mathrm{BD}$, and $\mathrm{CD}$; for 2 rats, the combination was $\mathrm{AD}$ ). In Test 3 , only one landmark was present, which differed across rats (A, B, and D were each used for 2 rats, and $C$ for 1 rat).

\section{Results and Discussion}

During training, all rats learned to find the platform very rapidly. An ANOVA of repeated measures showed that the days factor was significant both for the escape time measure $[F(7,55)=16.728, p<.01]$ and for the distance measure $[F(7,55)=27.558, p<.01]$. Partial comparisons showed that Day 1 differed from all the rest $(p<.05)$, among which we did not observe significant differences.

Figure 4 shows the mean time spent and the mean distance covered in Area PT versus Areas RI, LE, and OP on test trials. For Test 1 , with four landmarks, ANOVAs revealed significant differences both in the times that the subjects spent in the four quadrants $[F(1,13)=22.73, p<$ $.01]$ and in the distances covered within them $[F(1,13)=$ $17.836, p<.01]$.

For Test 2, with two landmarks, ANOVAs similarly revealed significant differences between the times that the subjects spent in the areas $[F(1,13)=13.287, p<.02]$ and in the distances covered $[F(1,13)=12.478, p<.02]$. In relation to the combination of cues tested $(\mathrm{AB}, \mathrm{AC}$, $\mathrm{AD}, \mathrm{BC}, \mathrm{BD}$, and $\mathrm{CD}$ ), Student's $t$ tests revealed no significant differences either in the time that was spent in the Area PT or in the distances covered. No differences were found when presence of Cue A (AB, AC, and AD) was compared with presence of other cues but absence of Cue $A(B C, B D$, and $C D)$. No differences were found when presence of the Cue $B(A B, B C$, and $B D)$ was com- 

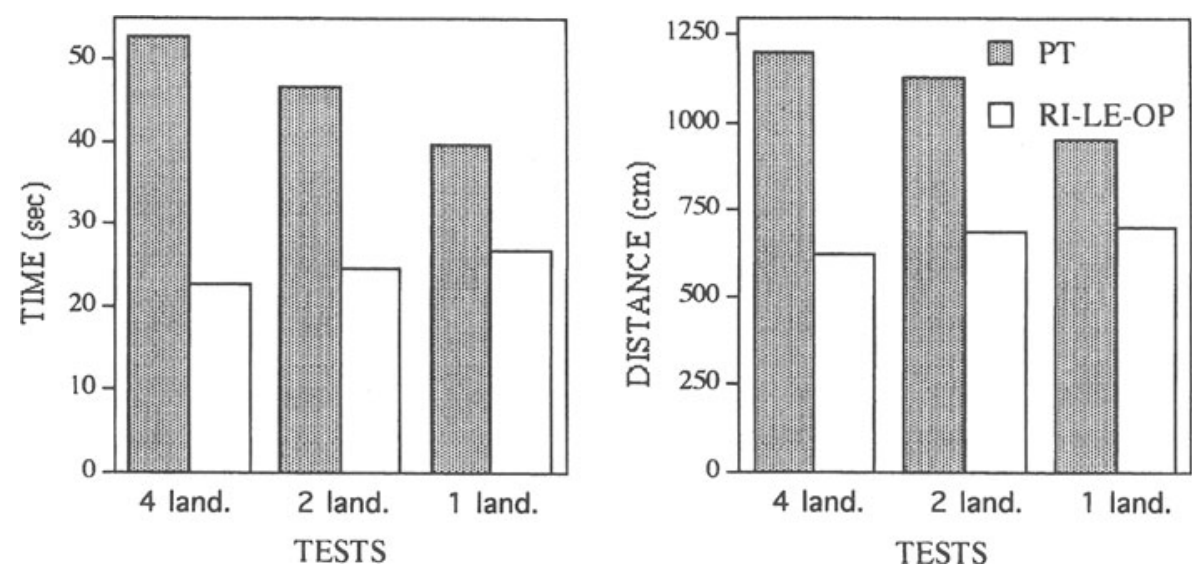

Figure 4. For the test phase of Experiment 2, mean time spent (left) and mean distance covered (right) by the subjects for the area in which the platform was located during the training (PT) and the other three areas (RI-LE-OP) in the presence of four landmarks, two landmarks, or one landmark.

pared with presence of other cues but absence of Cue B ( $A C, A D$, and $C D)$. No differences were found when presence of Cue $C(A C, B C$, and $C D)$ was compared with presence of other cues but absence of Cue $C(A B, A D$, and $B D)$. Also, there were no differences when presence of Cue $\mathrm{D}(\mathrm{AD}, \mathrm{BD}$, and $\mathrm{CD})$ was compared with presence of other cues but absence of Cue $\mathrm{D}(\mathrm{AB}, \mathrm{AC}$, and $\mathrm{BC})$.

For Test 3, with one landmark, no significant differences were found either in the times that the subjects spent in the areas $[F(1,13)=2.691, p=.1521]$ or in the distances covered $[F(1,13)=2.821, p=.144]$.

The main new result of Experiment 2 was the finding that, when the animals were tested with only two of the original four landmarks, they continued to show good performance, and this performance was independent of specific cues or combinations of cues. This extends the results previously found in other experimental conditions, such as the T-maze (O'Keefe \& Conway, 1978) and the radial maze (Chamizo et al., 1985). Thus, as in these other situations, animals trained in a water maze store redundant information that allows good performance even when significant changes occur in this environment. The results of Test 3 show that the rats required at least two landmarks to locate the platform. It should be noted that, in training, the landmarks and platform were rotated, with respect to the room, between each trial, in order to ensure that the rats used the defined landmarks, rather than any inadvertently remaining static room cues.

The present results are very consistent with those of Rodrigo, Chamizo, McLaren, and Mackintosh (1997, Experiment 1). Their rats could find an unseen platform in a swimming pool when the only way of determining its location was by reference to three or four specific external landmarks. When most of their initial training involved learning to swim to the platform, any two of these landmarks were sufficient for its accurate location. When only a single landmark was present, however, they showed a random-level performance on the test.

\section{EXPERIMENT 3}

A possible problem with our Experiment 2 and with Experiment 1 of Rodrigo et al. (1997) is that the test procedure confounded the number of landmarks with the order in which the different tests were given. Thus, it is possible that by the time that the subjects were tested with a single landmark some extinction of their original learning had taken place. Experiment 3 was undertaken to check the results of Experiment 2 using a different sequence of tests. Thus, to control for extinction during test phase, two landmarks were present for the first and fourth test and only one landmark for the second and third tests. If changes in performance over tests are determined by the number of landmarks available, test performance will be more accurate for the first and fourth tests than for the second and third tests. In addition, we were concerned that the subjects in Experiment 2 displayed a preference, even if not significant, for Area PT with one landmark.

\section{Method}

Subjects and Apparatus. The subjects were 7 Long-Evans rats ( 3 males and 4 females) 3 months old at the beginning of the experiment and experimentally naive. The landmarks used this time were the following: (A) a fixed light (a lamp, $15 \mathrm{~cm}$ in diameter and $20 \mathrm{~cm}$ high, with a $40-\mathrm{W}$ bulb); (B) a round diverse-colored balloon, $35 \mathrm{~cm}$ in diameter; $(C)$ an intermittent light that produced strong flashing signals. The location to the platform was between Cue B and Cue $\mathrm{C}$.

Procedure. As in Experiment 2, there were seven training sessions followed by four test sessions, with a test trial each day, each preceded by four retraining trials. The first test used two landmarks ( 3 rats were tested with the combination $\mathrm{AB}, 2$ with $\mathrm{BC}$, and 2 with $A C)$. The second test used only one landmark ( 3 rats were tested with A, 2 with B, and 2 with C). For each rat, the third test was identical to the second, and the fourth test was identical to the first.

\section{Results and Discussion}

During training, all 7 rats learned to find the platform very rapidly. An ANOVA of repeated measures showed 

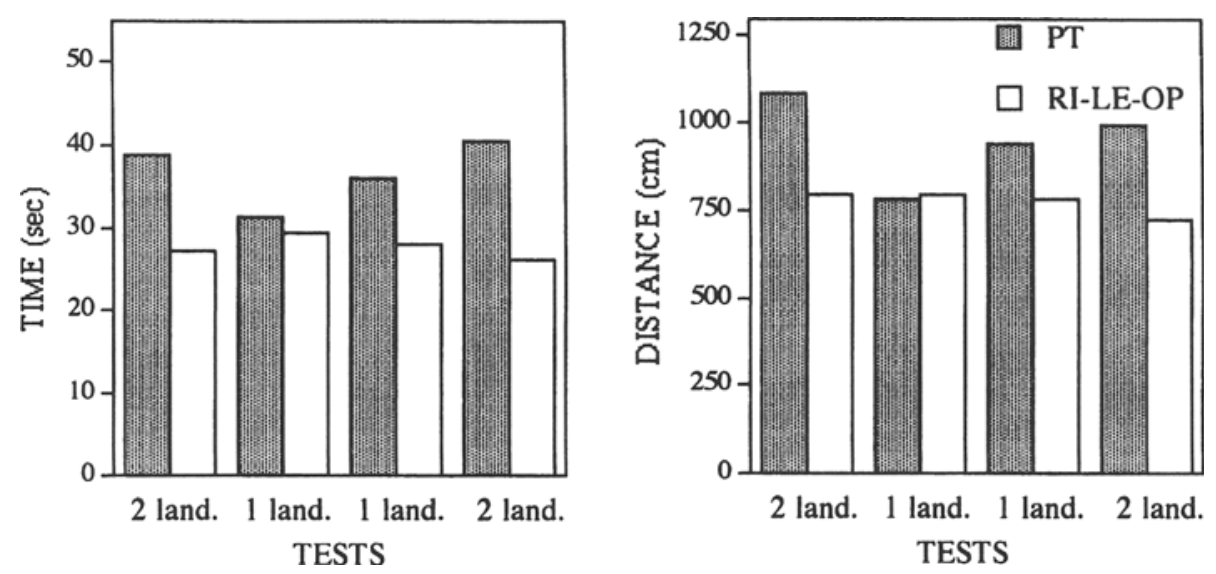

Figure 5. For the test phase of Experiment 3, mean time spent (left) and mean distance covered (right) by the subjects for the area in which the platform was located during the training (PT) and the other three areas (RI-LE-OP) in the presence of two landmarks or one landmark.

that the days factor was significant on both the escape time measure $[F(6,36)=12.504, p<.01]$ and on the distance measure $[F(6,36)=13.390, p<.01]$. Partial comparisons showed that, on both measures, Days 1 and 2 differed from all the rest $(p<.05)$, among which there were no significant differences.

Figure 5 shows the mean time and mean distance covered in Area PT versus Areas RI, LE, and OP during the test trials. ANOVAs of the data from the first test, with two landmarks, showed that the subjects spent significantly more time in Area PT than in the other areas $[F(1,13)=32.161, p<.01]$ and covered a greater distance in PT $[F(1,13)=41.293, p<.01]$. A similar analysis of the data from Tests 2 and 3 , with one landmark, failed to detect any significant differences either in the time that the subjects spent in the areas ( $p s>.10)$ or in the distance covered $(p s>10)$. Finally, the same ANOVA applied to the data from Test 4, with two landmarks, showed that, as for Test 1 , the subjects spent more time in Area PT $[F(1,13)=21.197, p<.01]$ and covered a greater distance in this area $[F(1,13)=11.249, p<.02]$.

The results from Experiment 3 confirm those from Experiment 2 and those from Rodrigo et al. (1997), and they indicate that the sequence of tests was not important. When two landmarks were present, the animals showed a good level of performance, as in Tests 1 and 4. However, when only one landmark was present, as in Tests 2 and 3, there was no indication that they were able to locate the position of the platform.

\section{GENERAL DISCUSSION}

The most marked difference between the procedure used here and that in previous research using the water maze (e.g., Morris, 1981) was that of surrounding the maze with black curtains and providing animals with a small number of cues to use as landmarks. The results from this set of three experiments show that the training procedure used under these conditions was an effective one for rats to learn to locate an invisible platform.

The results of Experiments 2 and 3 indicate that, having learned the location of a goal in an environment, animals can continue to locate this goal even when important changes are made to the environment. This supports the hypothesis that a cognitive map stores redundant information, thus allowing considerable flexibility (Chamizo et al., 1985; O'Keefe \& Conway, 1978). Cognitive representations of this kind give animals a powerful system of orientation that is able to cope with unexpected changes in their environment.

Previous research has examined other factors that affect performance in the water maze: for example, the relative familiarity of the different exit points, the light conditions during different phases of an escape trial, the withholding of visual access to a part of the cues in the experimental room, and the limit of physical access to a part of the pool (e.g., Devan, Blank, \& Petri, 1992; Sutherland, Chew, Baker, \& Linggard, 1987). The contribution of the present study is to show that animals can utilize the information stored in the form of a cognitive map to locate a goal when changes are produced in the environment represented. The results of Experiments 2 and 3 suggest that when animals have only a single landmark, they are unable to locate the position that the invisible platform occupied previously. However, when two or more objects are present with those on test, the animals showed a good level of performance. These results - excluding the possible interference of strange cues affecting the location of the goal, and knowing that the sequence of tests does not affect the results-lead us to conclude, as did Rodrigo et al. (in press), that the minimum information that they must have to locate a goal seems to be at least two landmarks. 


\section{REFERENCES}

Chamizo, V. D., Sterio, D., \& Mackintosh, N. J. (1985). Blocking and overshadowing between intra-maze and extra-maze cues: A test of independence of local and guidance learning. Quarterly Journal of Experimental Psychology, 37B, 235-253.

Devan, B. D., Blank, G. S., \& Petri, H. L. (1992). Place navigation in the Morris water task: Effects of reduced platform interval lighting and pseudorandom platform positioning. Psychobiology, 20, 120-126.

Hull, C. L. (1943). Principles of behavior. New York: Appleton-CenturyCrofts.

MoRRIs, R. G. M. (1981). Spatial localization does not require the presence of local cues. Learning \& Motivation, 12, 239-260.

O'KeEFE, J., \& CONWAY, D. H. (1978). Hippocampus place units in the freely moving rat: Why they fire where they fire. Experimental Brain Research, 31, 573-590.

O'KeEFE, J., \& NADEL, L. (1978). The hippocampus as a cognitive map. Oxford: Oxford University Press, Clarendon Press.
RestLe, F. (1957). Discrimination of cues in mazes: A resolution of the "place-vs.-response" question. Psychological Review, 64, 217-228.

Rodrigo, T., Chamizo, V. D., McLaren, I. P. L., \& Mackintosh, N. J. (1997). Blocking in the spatial domain. Journal of Experimental Psychology: Animal Behavior Processes, 23, 110-118.

Sutherland, R. J., Chew, G. L., Baker, J. C., \& Linggard, R. C. (1987). Some limitations on the use of distal cues in place navigation by rats. Psychobiology, 15, 48-57.

Tolman, E. C. (1932). Purposive behavior in animals and men. New York: Century.

Tolman, E. C. (1948). Cognitive map in rats and men. Psychological Review, 55, 189-209.

(Manuscript received April 18, 1996;

revision accepted for publication December 2, 1997.) 